\title{
Pharyngoconjunctival Fever
}

National Cancer Institute

\section{Source}

National Cancer Institute. Pharyngoconjunctival Fever. NCI Thesaurus. Code C34924.

A condition characterized by fever, conjunctivitis, and pharyng itis resulting from infection by adenovirus. 